\title{
Anisotropic In distribution in InGaN core-shell nanowires
}

\author{
C. Leclere, ${ }^{1}$ N. A. Katcho, ${ }^{2}$ G. Tourbot,${ }^{3}$ B. Daudin, ${ }^{3}$ M. G. Proietti,${ }^{4}$ and H. Renevier ${ }^{1, a)}$ \\ ${ }^{1}$ Laboratoire des Matériaux et du Génie Physique, Grenoble INP - Minatec, Grenoble, France \\ ${ }^{2}$ Liten, CEA-Grenoble, 17 rue des martyrs, 38054 Grenoble, France \\ ${ }^{3}$ CEA-CNRS group $\ll$ Nanophysique et Semiconducteurs $\gg$, Université Joseph Fourier and CEA Grenoble, \\ INAC, SP2M, 17 rue des Martyrs, 38054 Grenoble, France \\ ${ }^{4}$ Departamento de Fisica de la Materia Condensada, Instituto de Ciencia de Materiales de Aragon, \\ CSIC - Universidad de Zaragoza, Zaragoza, Spain
}

(Received 6 February 2014; accepted 23 June 2014; published online 7 July 2014)

\begin{abstract}
In this work, we investigate the local atomic structure of defect-free homogeneous and self-organized core-shell structure nanowires by means of X-ray Absorption Fine Structure (XAFS) Spectroscopy at the In $L_{I I I}$ and $K$ edges and Multiwavelength Anomalous Diffraction. The results are interpreted by comparison of the experimental data with X-ray absorption calculations carried out with ab initio structural models. Extended-XAFS data analysis at In K-edge shows an anisotropic In distribution in the second nearest neighbors pointing out to a deviation from randomness in In distribution for the core-shell sample. (C) 2014 AIP Publishing LLC.
\end{abstract}

[http://dx.doi.org/10.1063/1.4886756]

\section{INTRODUCTION}

In recent years, much progress has been made in understanding the relationships between the optical and structural properties of InGaN alloys but many important aspects remain unclear. The current debate focuses on the mechanisms responsible for the high radiative efficiency of InGaN Quantum Wells (QWs) in GaN despite the huge number of structural defects. It is generally assigned to exciton localization, which prevents carriers from undergoing non-radiative recombination at the structural defects, in particular, dislocations. Localization of holes has been demonstrated experimentally by Chichibu et al., ${ }^{1}$ who proposed a hole localization process, associated with atomic condensates of In-N. Such a mechanism could be associated to a deviation from InGaN alloy randomness and to the hypothetical formation of In-N-In chains or InN clusters of a few In atoms. To date, only two experimental observations at the atomic scale consistent with a kind of weak phase separation or In aggregation have been found, one by Kachkanov et al.,,3 and the second by Miyanaga et $a{ }^{4}{ }^{4}$ The existence of this kind of composition fluctuations has been discussed recently from a theoretical point of view by Zunger et $a l^{5,6}$ The theory predicts that In clustering occurs when dislocations and/or grain boundaries are present (incoherent alloy). If these extended defects are absent (coherent alloy), the elastic strain could cause In and Ga ordering. As a matter of fact, tiny structural variations in the atomic local environment, the exact nature of which is still to be established, can produce strong effects on optical properties, making InGaN materials virtually insensitive to the presence of extended defects as, for instance, dislocations.

\section{SAMPLES}

In this work, we have performed detailed studies of InGaN nanowires (NWs), which permit the investigation of

${ }^{a}$ Hubert.Renevier@grenoble-inp.fr
InGaN regions free of extended defects (i.e., they are model coherent alloys ${ }^{5,6}$ ). As shown recently by Tourbot et al., different morphologies depending on growth conditions can be obtained: NWs with the spontaneous formation of a coreshell structure, in which In is concentrated along the axial direction, and NWs with homogeneous In distribution. In case of the core-shell structure, the NWs are coherent with the GaN base NWs, whereas in the case of the homogeneous $\mathrm{NWs}$, dislocations are only present at the GaN interface (misfit dislocations), allowing the InGaN region to be dislocation free. ${ }^{7}$ To investigate the short range atomic structure of these two systems, we have combined Multiwavelength Anomalous Diffraction (MAD) and X-ray Absorption Fine Structure (XAFS) spectroscopy with ab initio calculations. MAD was used for determining the actual core and shell In content. XAFS has given the local atomic structure and $a b$ initio calculations were used to fully interpret the XAFS results. The samples were grown by Plasma Assisted Molecular Beam Epitaxy in the CEA-CNRS group (Grenoble) on a GaN NW base grown on $\mathrm{Si}(111)$ substrate. The growth direction was along the c-axis ([001] direction). Details about growth and photoluminescence (PL) characterization are published elsewhere. ${ }^{7}$ Two different InGaN NW samples corresponding to two different morphologies have been investigated: (a) sample $1, \operatorname{In}_{x} G a_{1-x N} \mathrm{NWs}$ with a nominal In content of $17 \%$. These NWs show a self-organized core-shell structure (Fig. 1(c)). Their PL spectra as a function of temperature exhibit a typical S-shape feature characteristic of carriers localization. (b) Sample 2, $\operatorname{In}_{x} G a_{1-x} N$ NWs with a nominal In content of $43 \%$. These NWs show homogeneous In distribution and wider PL spectra, compared to sample 1 .

\section{MULTIWAVELENGTH ANOMALOUS DIFFRACTION}

We report, in Fig. 1, X-ray diffraction Reciprocal Space Maps (RSMs) which put in evidence the different 

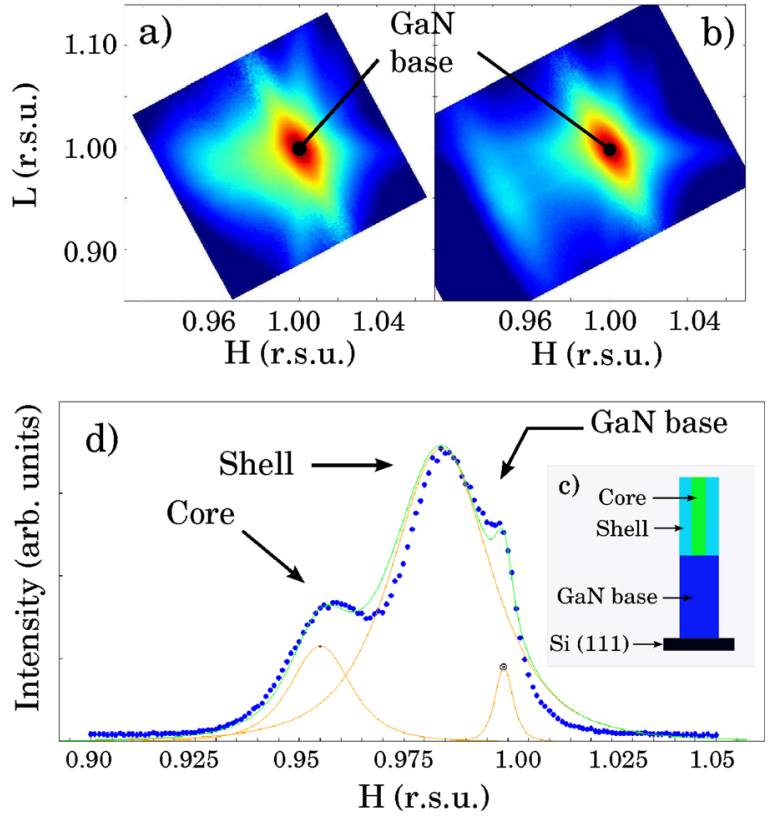

FIG. 1. (a) X-ray Reciprocal Space Maps close to the $10 \overline{1} 1 \mathrm{GaN}$ reflection for elastically strained core-shell NWs (sample 1) and (b) relaxed NWs (sample 2). (c) Sketch of sample 1. (d) $\mathrm{H}$-scan in grazing incidence of sample 1 with an X-ray beam energy $E=10600 \mathrm{eV}$ and an incidence angle $\alpha_{i}=0.08^{\circ}$, close to the $11 \overline{2} 0 \mathrm{GaN}$ reflection. Reciprocal space units (r.s.u.) refer to relaxed $\mathrm{GaN}$ parameters $(a=3.185 \AA$ and $c=5.189 \AA)$. The fit curves (solid lines) represent the diffraction contributions of the core, shell, and GaN NW base to the diffracted intensity.

morphologies of samples 1 and 2, and allow the determination of their average lattice parameters and strain state. RSMs were recorded with an X-ray energy of $10200 \mathrm{eV}$, close to $10 \overline{1} 1 \mathrm{Bragg}$ reflection of GaN, with a Vantec ${ }^{\mathrm{TM}}$ linear detector. The measurements were carried out at beamline BM2 at ESRF in Grenoble (France). In Figs. 1(a) and 1(b), the high intensity spots, at $H=L=1$, correspond to the relaxed $\mathrm{GaN}$ base of the NWs, as sketched in Fig. 1(c). For sample 1 (Fig. 1(a)), at $L=0.985$ we observe scattered intensity that extends to low- $H$ values, which corresponds to the core and shell regions. This is shown in more detail in Fig. 1(d) by a $H$-scan recorded in grazing incidence close to $11 \overline{2} 0$ reflection with a X-ray beam energy equal to 10600 $\mathrm{eV}$, i.e., an energy above the $\mathrm{Ga} \mathrm{K}$-edge $(10367 \mathrm{eV})$. Here, the GaN contribution is strongly reduced due to self absorption in the wire region and one can better distinguish between the core and shell regions. Figure 1(b) shows a RSM for sample 2. The spot at $H=0.94$ and $L=0.95$ values corresponds to relaxed InGaN with a homogeneous composition profile. As can be seen in the RSMs, one can select $H$ and $L$ values that correspond to the shell and core regions of sample 1 and to the relaxed homogeneous InGaN NWs of sample 2 .

To determine the actual composition of the different InGaN regions selected by diffraction, we performed MAD experiments. ${ }^{9}$ For that purpose we measured for samples 1 and 2, diffracted intensity spectra as a function of X-ray beam energy close to the Ga K-edge $(10367 \mathrm{eV})$. Fig. 2 shows for samples 1 and 2, diffracted intensity spectra corrected for Ga-fluorescence yield, as a function of X-ray beam energy. The spectra were measured at reciprocal space

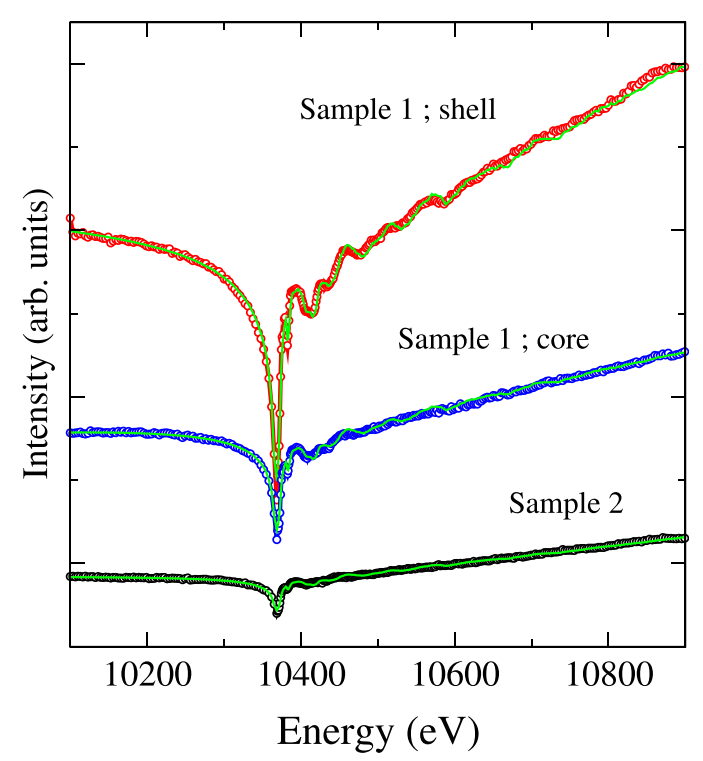

FIG. 2. Energy scan across the Ga K-edge $(10367 \mathrm{eV})$ at fixed $H=0.955$, $K=0, L=0.982$ (core) and $H=0.980, K=0, L=0.983$ (shell) positions in the reciprocal space for sample 1 and $H=0.94, K=0, L=0.95$ for sample 2 . The spectra are corrected for fluorescence. Both experimental (open symbols) and best fit curves (solid lines) are shown. The best fit In contents are reported in Table I.

coordinates $H=0.955, K=0, L=0.982$ and $H=0.980$, $K=0, L=0.983$, which correspond to the core and shell regions of sample 1 , respectively, and at $H=0.940, K=0$, $L=0.950$ for sample 2 . The cusps of the diffracted intensity spectra were fitted by calculating the diffraction of a homogeneous $\operatorname{In}_{x} \mathrm{Ga}_{1-x} \mathrm{~N}$ alloy with the wurtzite structure. The fit procedure is described in detail in previous publications. ${ }^{9,10}$ The Ga anomalous scattering factor $f^{\prime \prime}$ was obtained from a fluorescence spectrum measured at reciprocal space coordinates close to those of diffracted intensity spectra. The correspondent $f^{\prime}$ factor was obtained by Kramers-Kronig transformation of $f^{\prime \prime}$. The fit parameters were a scale factor, the detector efficiency as a function of the energy, and the In content $x$.

The XRD and MAD results on samples 1 and 2 are reported in Table I. For sample 1, they show that the core and shell regions have very different In contents, i.e., 33\% and $6 \%$, respectively. The In-enriched core (33\% is much larger than the nominal value of $17 \%$ ) is compressively strained along $\mathrm{c}$ axis, due to the lattice mismatch with the Ga-rich shell $(6 \%)$, which undergoes in turn tensile strain

TABLE I. Structural parameters (lattice parameters and In content $\left(x_{I n}\right)$ ) of samples 1 and 2 as determined by XRD and MAD experiments. Lattice parameters of GaN NWs base are reported for comparison. HKL (MAD) refers to the reciprocal coordinates at which the energy-scan has been performed for determining the In content. All $H, K$, and $L$ values are in reciprocal lattice units (r.s.u).

\begin{tabular}{lcccccr}
\hline \hline Sample & $H$ & $\mathrm{a}(\AA)$ & $L$ & $\mathrm{c}(\AA)$ & $H K L(\mathrm{MAD})$ & $x_{\text {In }}(\%)$ \\
\hline Sample 1 core & 0.956 & 3.33 & 0.985 & 5.267 & $0.955,0,0.982$ & $33 \pm 1$ \\
Sample 1 shell & 0.985 & 3.23 & 0.985 & 5.267 & $0.980,0,0.983$ & $6 \pm 1$ \\
Sample 2 & 0.946 & 3.37 & 0.952 & 5.44 & $0.940,0,0.950$ & $46 \pm 1$ \\
GaN NWs base & 1 & 3.18 & 1 & 5.189 & $1,0,1$ & $0 \pm 1$ \\
\hline \hline
\end{tabular}


along the same axis. As a result of the presence of strain, Vegard's law cannot be used for determining the composition. Conversely, for sample 2 In content (46\%) and lattice parameters are in agreement with Vegard's law showing that the InGaN alloy is relaxed.

\section{X-RAY ABSORPTION FINE STRUCTURE}

To gain information on the local atomic short range order (SRO) structure of sample 1 (core) and sample 2, we measured XAFS spectra at the $L_{I I I}$ and $\mathrm{K}$ edges of In. A XAFS spectrum is classically divided into a near-edge region (X-ray Absorption Near Edge Structure) spanning from the edge up to about $50 \mathrm{eV}$ above the edge and an Extended XAFS (EXAFS) region extending from XANES up to about $1000 \mathrm{eV}$ above the edge. In the EXAFS region, Single Scattering (SS) events, involving only two atoms, dominate and the analysis of the oscillations can be carried out according to a path development formalism, which allows a fitting procedure, and gives direct information on structural parameters as interatomic distances and coordination numbers. The analysis of the near edge region (XANES) is more complicated since Multiple Scattering (MS) events, involving three or more atoms, are much more favored and the paths development approach is no longer valid. Nevertheless, XANES is very sensitive due to MS to the local atomic arrangement and state-of-the art ab initio calculations provide accessible insight to XANES spectra that can be interpreted by comparison with simulations of different models. XANES spectra in fluorescence mode at the In $L_{I I I}$ edge $(3730 \mathrm{eV})$ of samples 1 and 2 were recorded at XAFS beamline 11.1 of Elettra Synchrotron in Trieste (Italy). The samples were mounted in vacuum with the surface normal oriented at $45^{\circ}$ with respect to the incoming beam direction. The fluorescence yield was recorded in the horizontal plane at $90^{\circ}$ with respect to the $\mathrm{X}$-ray beam direction, with a one element Peltier-cooled solid state detector.

\section{A. X-ray absorption near edge structure}

The XANES spectra are shown in Fig. 3 for samples 1 and 2. One can clearly see that the spectra have different shapes. In particular, the XANES spectrum of sample 1 shows two features at $3795 \mathrm{eV}$ (A) and $3876 \mathrm{eV}$ (B), which are smeared out on the spectrum of sample 2. So far, we must note that for sample 1 the XAFS spectrum is the weighted average of the shell and core regions yields, with an In concentration of $6 \%$ and $33 \%$, respectively, and a core/ shell volume ratio (1/2.5) which was determined from grazing incidence X-ray diffraction profile (see Fig. 1(d)).

EXAFS spectra of samples 1 and 2 were recorded at the In K-edge $(27940 \mathrm{eV})$, at room temperature, in fluorescence mode by using a Canberra ${ }^{\mathrm{TM}} 30$ elements Ge detector, at beamline BM30B of ESRF. The fluorescence yield was detected in the horizontal plane at $90^{\circ}$ with respect to the $\mathrm{X}$-ray beam direction. The spectra were recorded by orienting the sample surface nearly parallel to the incident beam (incidence angle equal to about $5^{\circ}$ ) and perpendicular to it (incidence angle equal to about $85^{\circ}$ ). In such a way, the X-ray photons polarization vector $\vec{\varepsilon}$ was nearly perpendicular

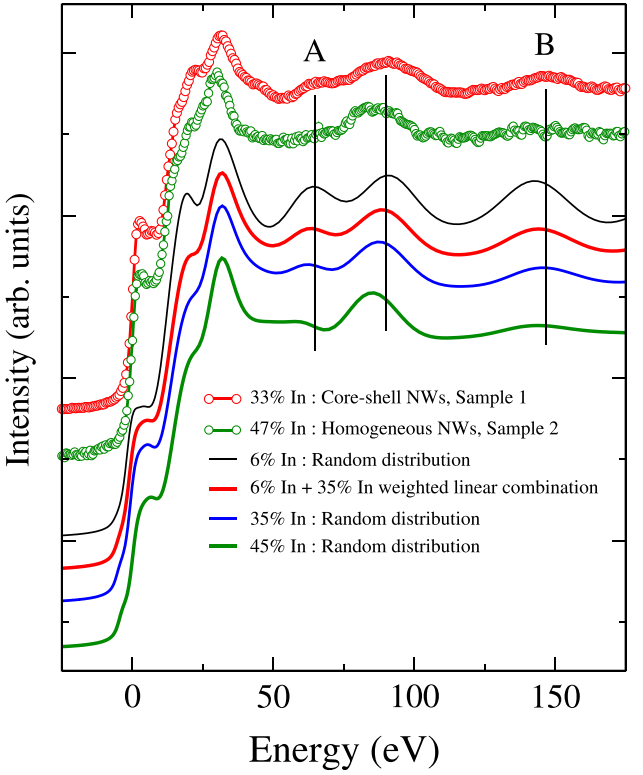

FIG. 3. Experimental XAFS spectra (open symbols) at In $L_{I I I}$ edge (3730 eV is the reference energy) for self-organized core-shell (sample 1) and relaxed NWs (sample 2). They are compared to simulated XAFS spectra (solid lines) that correspond to structural models relaxed by ab initio calculations for different In content. From upper to lower curve: $6 \%$ In random distribution (black curve), 35\% In random distribution (blue curve), $45 \%$ In random distribution (green curve). A and B are typical features for low In content (6\% for instance). The red curve is the linear combination of the black and blue curves taking into account the core/shell volume ratio (1/2.5).

and parallel to the sample surface, respectively. The background subtracted EXAFS spectra are shown in Fig. 5. The reason for measuring EXAFS with parallel and perpendicular polarisation is to exploit the strong anisotropy of the EXAFS probe at the K-edge. ${ }^{11}$ Indeed, in the SS approximation, the EXAFS spectrum can be expressed by ${ }^{12}$

$$
\chi(k)=S_{0}^{2} \sum_{j=\text { shells }} \frac{N_{j} f_{j}\left(k, r_{j}\right)}{k r_{j}^{2}} e^{-2 k^{2} \sigma_{j}^{2}} \sin \left(2 k r_{j}+\varphi_{j}(k)+2 \delta\right),
$$

where $r_{j}, N_{j}$, and $\sigma_{j}$ are the interatomic distance, the effective coordination numbers, and the bond-length disorder (static and dynamic Debye-Waller factors), $S_{0}^{2}$ is the EXAFS amplitude reduction factor due to multielectrons excitation channels, $\varphi_{j}$ and $f_{j}$ are the phase shift and the backscattering amplitude of atoms in shell $j, \delta$ is the phase shift due to the central atom and $k$ is the photoelectron wavenumber. In expression (1), $N_{j}$ is defined as

$$
N_{j}=\sum_{i} 3\left(\cos ^{2} \theta_{i}\right)
$$

where $\theta_{i}$ is the angle between the electric field vector of the incoming $x$-ray beam and the direction of the photoelectron scattering path $i$ in shell $j$. For polycrystalline samples, the sum over all the randomly oriented scattering paths gives the coordination number of the atomic shell of radius $r_{j}$. In monocrystalline InGaN samples, which crystallise in the hexagonal space group $P 6_{3} m c$ (wurtzite), the In/Ga atoms belonging to the II coordination shell (Next Nearest Neighbor, NNN) are 12: 6 in the growth plane (in-plane) and 6 out of growth plane (out-of plane). The contribution of 
in-plane (resp. out-of-plane) atoms is 9 (resp. 3) for parallel polarization and 0 (resp. 12) for perpendicular polarization of the beam. In this way, simultaneous fitting of spectra obtained with parallel and perpendicular polarization allows us to determine the $a$ and $c$ lattice parameters and the $\mathrm{In} / \mathrm{Ga}$ ratio, i.e., In concentration, in-plane and out-of-plane, and to detect the presence of lattice deformations due to strain and of anisotropies in composition produced by deviations from random atomic distribution in the alloy.

\section{B. Extended X-ray absorption fine structure}

The EXAFS signals were calculated for a cluster of InN composed by 50 atoms, with a radius of $5.70 \AA$. To include $\mathrm{Ga}$ atoms, we calculated the same cluster substituting all the In atoms, but the central absorber, with $\mathrm{Ga}$. In the fit procedure, the scattering paths of both clusters, involving $\operatorname{In}(\mathrm{Ga})$ as scatterers, were included and weighted by a factor $x$ for In and $(1-x)$ for Ga. In such a way, $x$ is refined and represents the In concentration. Theoretical amplitudes and phase shifts were calculated $a b$ initio by FEFF8 code $^{13}$ taking into account the beam polarization vector, $\vec{\varepsilon}$, directed along the [110] and [001] crystallographic directions, corresponding to the parallel and perpendicular orientations with respect to the (0001) surface plane. Scattering potentials were calculated in the muffin-tin approximation in a self-consistent way, with a Hedin-Lundqvist approximation for exchange and inelastic losses.

The theoretical EXAFS signal is composed by a huge number of SS and MS paths due to the high number of atoms belonging to the cluster and to the polarization dependence of $\chi(k)$. In the fitting procedure, we took into account a limited number of them that represent the main contribution to the EXAFS signal in the real space region ( $\mathrm{R}$ region)up to 4.5 ̊. We included 5 SS paths: In-N (I shell), In-In(Ga) (II shell), In-N (III shell), and In-N (IV shell). The contribution of the IV In-N shell does not depend strongly on polarisation and its contribution is quite weak and broad, but it sums up to the II $\operatorname{In}(\mathrm{Ga})$ shell contribution. We also included 3 MS triangular paths (In-N-N, In-N-In, and In-N-Ga). These MS paths give a weaker contribution than SS paths but they improve the fit quality and II shell distances determination (as shown for analogous compounds as $\mathrm{GaN}$ or $\mathrm{InGaAs}^{12}$ ). The fits parameters were, $E_{0}, S_{0}^{2}$, DW factors for each path, $d(\operatorname{In}-N)$ in I shell, $d_{i n}(I n-I n), d_{i n}(I n-G a), d_{\text {out }}(I n-I n)$, and $d_{\text {out }}(\mathrm{In}-\mathrm{Ga})$ in II shell, $d(\mathrm{In}-N)$ in III shell, and In concentration, $x_{i n}$ and $x_{\text {out }}$, where in (resp. out) means in-plane (resp. out-of-plane). The average value of $d_{i n}(I n-I n)$ and $d_{i n}(I n-G a)$ in II shell corresponds to the lattice parameter $a$. The interatomic distances $d_{\text {out }}(I n-I n)$ and $d_{\text {out }}(I n-G a)$ in II shell are a combination of $d_{i n}(I n-I n)$ and $d_{i n}(I n-G a)$ in II shell and $c$, according to hexagonal symmetry

$$
d_{\text {out }}=\sqrt{\frac{1}{3} d_{\text {in }}^{2}+\frac{1}{4} c^{2}} .
$$

Also, the MS paths lengths and $d(\operatorname{In}-N)$ (IV shell) were defined in the fit as a combination of $a$ and $c$ according to the hexagonal symmetry. $S_{0}^{2}$ was refined and found to be 0.9 . The I neighbors distance $d(\operatorname{In}-N)$ was refined to a common value for perpendicular and parallel polarisation. A splitting would be expected due to the tetrahedric bond orientation in the wurtzite structure for which one of the four $\mathrm{N}$ atoms lays along [001] and the other three are close to $a-b$ plane; nevertheless, it is small, even in the presence of strain (about $0.007 \AA) .{ }^{14}$ It is lower than the error we have on the best fit values for the first shell distance $(0.01 \AA)$. The fits were performed simultaneously for the two polarizations to improve fit statistics.

The background subtracted EXAFS spectra for samples 1 and 2 are shown in Fig. 5, together with the corresponding best fit curves. Background subtraction in the EXAFS region was performed by AUTOBK code implemented by the ATHENA graphical interface. ${ }^{15}$ EXAFS data analysis was performed by using the IFEFFIT ${ }^{8}$ code implemented by the ARTEMIS interface. ${ }^{15}$ The best fit results are shown in Table II.

For sample 1, we find an In concentration in the II coordination shell that is different for the two polarization directions. It is equal to $21 \%$ for the out-of-plane polarisation and $31 \%$ for the in-plane polarization. Therefore, the EXAFS average In concentration is $(0.31+0.21) / 2=0.26$ (the number of NNN is the same for in-plane and out-of-plane directions), the conditional probability to find an In atom in the NN environment of a In atom. This value is very close to the value calculated with the In concentrations found by MAD knowing the core/shell volume ratio and assuming a random distribution. Indium atoms in the core contribute to the XAFS signal with a weight equal to $0.33 /(0.33+2.5 \times 0.06)=0.7$; therefore, the XAFS average In concentration in the II shell would be equal to $0.7 \times 0.33+0.3 \times 0.06=0.25$. Those values, 0.26 and 0.25 , are much higher than the average In content found by diffraction (MAD): $\bar{x}_{I n}^{M A D}=(0.33+2.5$ $\times 0.06) / 3.5=0.14$. This is an expected result showing that In atoms have segregated to form an In rich core. The segregation can be quantified by calculating the Warren-Cowley SRO parameters, ${ }^{16}$ defined as $\alpha_{j}=1-P_{j}^{(A) B} / x_{B}$, where $P_{j}^{(A) B}$ represents the conditional probability of finding an atom of type $B(\mathrm{Ga})$ in the $j$ th coordination shell of an atom of type $A$ (In) and $x_{B}$ is the concentration of atom $B$. The

TABLE II. Nearest neighbor and next nearest neighbor distances and NNN In contents for the samples studied, in the growth direction (out-of-plane) and in the growth plane (in-plane), obtained by best fit of EXAFS data by using IFEFFIT ${ }^{8}$ code. $^{2}$

\begin{tabular}{|c|c|c|c|c|c|c|c|c|}
\hline Sample & d(In-N) (̊) & $d_{i n}(\mathrm{In}-\mathrm{In})(\AA)$ & $d_{i n}(\mathrm{In}-\mathrm{Ga})(\AA)$ & $d_{\text {out }}(\operatorname{In}-\mathrm{In})(\AA)$ & $d_{\text {out }}(\mathrm{In}-\mathrm{Ga})(\AA)$ & $c(\AA)$ & $x_{I n, i n}(\%)$ & $x_{\text {In,out }}(\%)$ \\
\hline Sample 1 & $2.11 \pm 0.01$ & $3.31 \pm 0.02$ & $3.28 \pm 0.02$ & $3.26 \pm 0.02$ & $3.25 \pm 0.02$ & $5.29 \pm 0.02$ & $31 \pm 4$ & $21 \pm 3$ \\
\hline Sample 2 & $2.13 \pm 0.01$ & $3.39 \pm 0.02$ & $3.34 \pm 0.02$ & $3.35 \pm 0.02$ & $3.33 \pm 0.02$ & $5.45 \pm 0.03$ & $54 \pm 8$ & $44 \pm 4$ \\
\hline GaN bulk & 1.95 & $3.18(\mathrm{Ga}-\mathrm{Ga})$ & $\ldots$ & $3.18(\mathrm{Ga}-\mathrm{Ga})$ & $\ldots$ & 5.189 & $\ldots$ & \\
\hline InN bulk & 2.15 & 3.54 & $\ldots$ & 3.54 & $\ldots$ & 5.708 & $\ldots$ & \\
\hline
\end{tabular}


sign of $\alpha$ indicates whether the central atom and atoms in the $j$ coordination shell tend to be of the same kind $(\alpha>0$, clustering) or tend to be of a different kind $(\alpha<0$, anticlustering or ordering). In the present case, $\alpha_{2}=1-P_{2}^{(I n) G a} / \bar{x}_{G a}^{M A D}$ $=0.14>0$. Note, as we know the In contents in the core and shell regions from MAD, it is convenient to follow up by defining the random case as being the random distribution of $6 \%$ (resp. 33\%) In in the shell (resp. in the core).

As mentioned above, the In concentration in II shell for sample 1 is higher in-plane (31\%) than in the growth direction $(21 \%)$. If the In distribution, both in the core and shell regions, was completely random, one should find the same value, i.e., $25 \%$. This indicates that, when considering the whole sample, the relative deviation from randomness is $+24 \%$ in-plane and $-16 \%$ out-of-plane. This anisotropic behaviour in the distribution of In-In pairs in the II coordination shell could suggest a tendency to In clustering in the growth plane and to In short range ordering in the growth direction. Unfortunately, as we cannot disentangle the shell and core contributions by EXAFS we cannot go further in detail concerning SRO in the core and shell separately.

Regarding sample 2, first, we find that the out-of-plane In concentration in II shell (44\%) is equal, within the experimental errors, to the average value found by MAD (46\%), showing that the out-of plane In distribution is random. Second, the in-plane In concentration is higher (54\%) than the MAD value of $46 \%$ corresponding to a relative deviation from randomness of $+17 \%$. This last result could suggest a similar behaviour as in sample 1 for the in-plane In distribution, but the effect is weaker and, in addition, the result about coordination numbers must be taken with caution because the EXAFS spectrum of sample 2 is affected by a low $\mathrm{S} / \mathrm{N}$ ratio leading to large experimental errors. So in this case we cannot state that a clear anisotropy is observed for sample 2, as confirmed by the ab initio simulation results reported in Sec. V.

We want to stress here on the combination of MAD and EXAFS data. MAD cannot give information about anisotropy at local scale, this is provided by EXAFS. On the other side, for a correct interpretation of the EXAFS results of Table II one needs to know the average In concentration determined in an independent way.

Regarding interatomic distances, for sample 1, the d(In$\mathrm{N})$ value is close to the value for bulk $\mathrm{InN}$, as expected for III-V semiconductors, in which bond distances do not follow the Vegard's law and stay close to the values of the correspondent binary compounds. ${ }^{17}$ The In-Ga(In) distances corresponding to the NNN atoms are quite close to each other and close to the values foreseen by the Vegard's law for alloys, as observed by other authors for InGaN. ${ }^{2-4}$ In addition, they are smaller in the growth direction (out-of-plane) than in the growth plane (in-plane). They are also smaller than the values corresponding to the relaxed alloy. This is in agreement with the presence of $c$-matching with the $\mathrm{Ga}$ enriched shell region, as shown by RSM. Regarding sample 2 , the values found for $a$ and $c$ are quite close to the value foreseen by the Vegard's law, in agreement with the relaxed nature of this sample as observed by RMS.

\section{AB INITIO CALCULATIONS}

XAFS data have been interpreted by simulation of $L_{I I I}$ and $K$ edges for a random In distribution in the core and shell regions. The input parameters of the structural models were the lattice parameters and compositions measured by diffraction (Table I). For sample 1, we generated two structural models to represent the core and the shell. As sample 2 is homogeneous, we generated only one model.

Initially, the atoms were located in the crystallographic sites of a perfect wurtzite structure, with a random distribution of In/Ga atoms. We used a $4 \times 4 \times 4$ supercell (256 atoms). Then, we performed an $a b$ initio energy minimization, at constant volume, to relax the atomic positions. The calculations were carried out using the Vienna $a b$ initio Simulation Package (VASP). ${ }^{18,19}$ They were performed in the generalized gradient approximation (GGA) using the PW91 functional of Perdew and Wang, with a $2 \times 2 \times 2 k$ point sampling and an energy cutoff of $350 \mathrm{eV}$.

Figure 4 shows the results of the structure relaxation for a random distribution with $33 \%$ (core, sample 1) and $46 \%$ In contents (sample 2). Then, with the structural model we performed $a b$ initio calculations of the XANES spectra at Indium $L_{I I I}$ edge by using the FDMNES code ${ }^{20}$ in the muffin-tin potential approximation. Calculations were performed at each $\mathrm{In}$ site of the $4 \times 4 \times 4 \mathrm{InGaN}$ supercell taking a $5 \AA$ radius sphere that includes up to seven coordination shells. The spectra, corresponding to each In atom in the $4 \times 4 \times 4$ supercell, were averaged out to get the overall XANES spectrum. Thermal disorder was taken into account by convolution with a gaussian corresponding to Debye Waller factors equal to $0.025 \AA^{-2}$. The results are shown in Fig. 3. With the same models we also calculated the EXAFS spectrum by using theoretical amplitudes and phase shifts generated by the FEFF8 code, in the paths development approximation. EXAFS spectra were calculated for each In absorber in the supercell, considering the neighbors atoms belonging to a $5 \AA$ radius sphere, and averaging out all the contributions. The results are shown in Fig. 5.

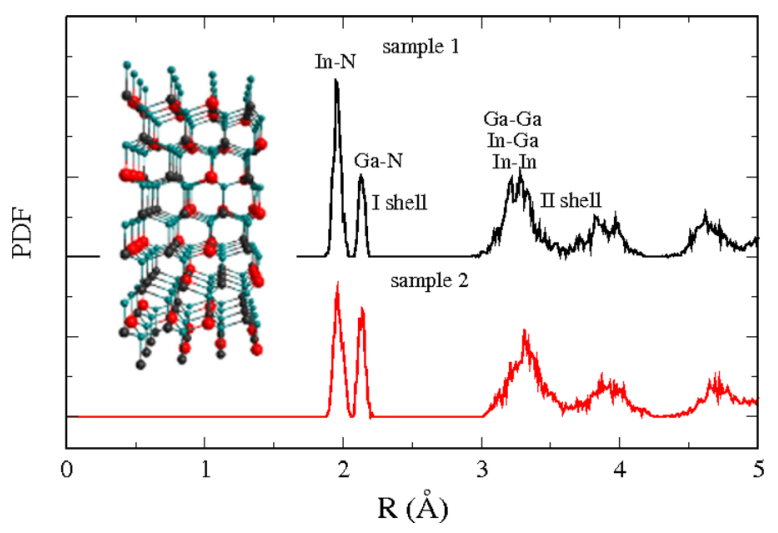

FIG. 4. Pair distribution function after $a b$ initio relaxation of the atomic positions in a $4 \times 4 \times 4$ InGaN supercell with $33 \%$ In (black curve) and $46 \%$ In content (red curve). In atoms are randomly distributed in both cases. Inset: Structural model shown along [110] direction for the 33\% In content. Red, black, and blue balls represent In, Ga, and $\mathrm{N}$ atoms, respectively. 


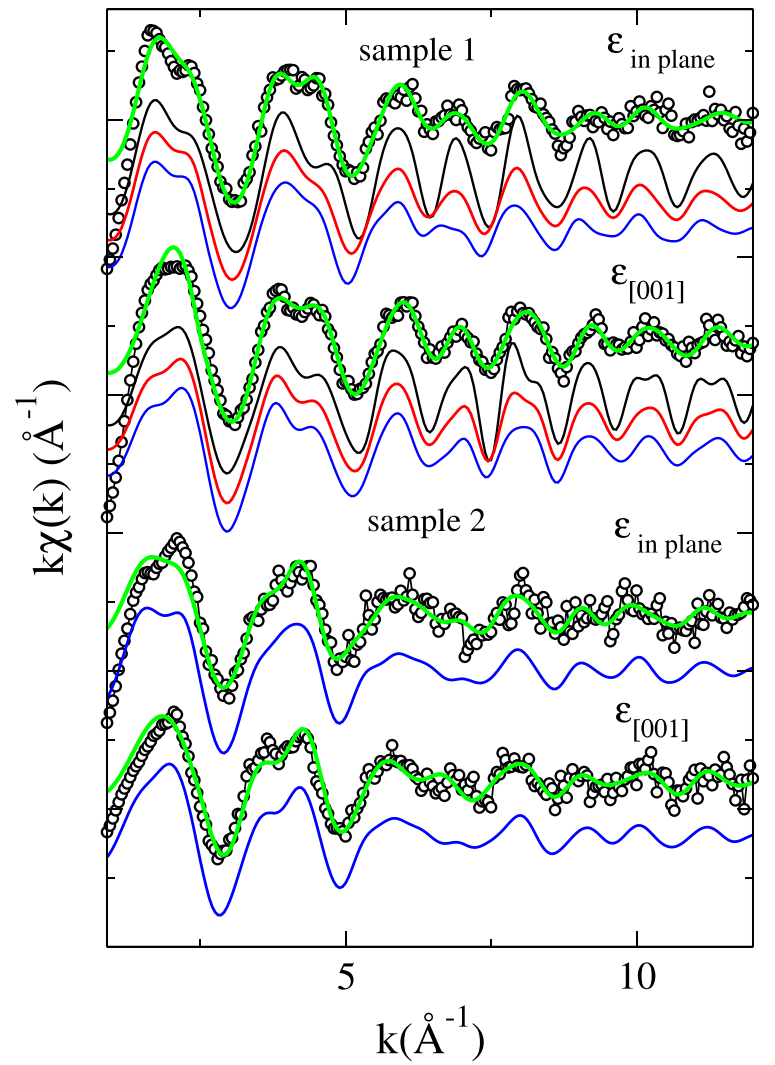

FIG. 5. Experimental EXAFS spectra (open symbols) with in-plane and outof-plane ([001]) X-ray beam polarizations, at In $K$ edge, for self-organized core-shell nanowires (sample 1, upper panel) and homogeneous nanowires (sample 2, lower panel). They are compared to best fit curves (green solid lines) and to simulated EXAFS spectra (solid lines shifted downwards). The latter correspond, for sample 1, to structural models relaxed by ab initio calculations for different In content, from upper to lower curve: 6\% In random distribution (black curve), 33\% In random distribution (blue curve). The red curve is the linear combination of the black and blue curves taking into account the core/shell volume ratio (1/2.5). For sample 2, simulated EXAFS spectra correspond to In random distribution (blue curve).

\section{DISCUSSION}

The results of XAFS calculations for random models are shown in Figs. 3 (XANES) and 5 (EXAFS) and compared to the experimental spectra of samples 1 and 2. Regarding XANES results (Fig. 3), one can see on the experimental spectra that features (A) and (B) are a signature of a low In concentration in the $\mathrm{Ga} / \mathrm{In}$ II shell around the In absorber. They are quite pronounced for sample 1 and completely smeared out for sample 2. Indeed, simulation curves obtained with $\mathrm{In} / \mathrm{Ga}$ random distribution model demonstrate that this is mainly due to the different average composition of the two samples. In Fig. 3, simulated XAFS spectra (solid lines) correspond to different In contents. From upper to lower curve: $6 \%$ In random distribution (black curve), 35\% In random distribution (blue curve), and $45 \%$ In random distribution (green curve). The red curve is the linear combination of the black and blue curves taking into account the core/shell volume ratio $(1 / 2.5$, i.e., a $70 \%$ contribution of the core). The experimental XAFS spectrum of sample 1 is well reproduced by the linear combination. It has to be noted that the sensitivity of XAFS at the $L_{I I I}$-edge to anisotropic atomic distribution is much weaker than in the case of $K$-edge due to the different amplitude polarisation factor dependence of the absorption process. Moreover, in our measurements the beam polarisation vector was oriented at $45^{\circ}$ with respect to the sample surface, i.e., close to the magic angle of $54.7^{\circ}$ for which XANES is equally sensitive to in-plane and out-ofplane atoms in II shell (NNN). ${ }^{11}$ Polarized EXAFS spectra measured at the In $K$-edge and the best fit curves (solid green line) are shown in Fig. 5 for samples 1 and 2. Fig. 5 also shows simulated polarized EXAFS spectra (solid lines) corresponding to random In distributions. The experimental [001]-polarised EXAFS spectrum of sample 1 is qualitatively well reproduced by the linear combination of EXAFS spectra corresponding to $6 \%$ and $33 \%$ In random distribution and taking into account the core/shell volume ratio. Instead, in the case of the in-plane-polarised XAFS the spectrum shape is not very well reproduced by the same linear combination, especially in the range from $3 \AA^{-1}$ to $4 \AA^{-1}$ where the simulation shows a different shape compared with experiment that corresponds to an In content lower than the experimental one. These results are in very good agreement with the polarised XAFS best fit results which give $31 \%$ in-plane and $21 \%$ out-of-plane with respect to $25 \%$ (random case). More details about the Short Range Order in the core and the shell should require additional experiments out of the scope of the present paper. In particular, one needs to know the conditional probability to find a In atoms in the II shell of an In atom either in the core or shell regions, to be compared to the actual average In contents given by MAD (i.e., 33\% and $6 \%$ for the core and the shell, respectively). This could be obtained from the Extended Diffraction Anomalous Fine Structure ${ }^{9,10}$ oscillations to be measured at the core and shell positions in the reciprocal space and at the In- and Ga K-edges. Regarding sample 2, in which the mismatch strain is fully relaxed, the experimental XAFS spectra (XANES, EXAFS) are well reproduced by the random distribution model (Figs. 3 and 5, solid green line and solid blue lines, respectively).

\section{CONCLUSION}

Our results show that XAFS and MAD data can be interpreted in a coherent way providing evidence of an anisotropic In distribution in InGaN core/shell nanowires. That is, there exists more In-In pairs in-plane than out-of-plane with respect to the random distribution. We also observe that this takes place in sample 1, which has a strained In enriched core and barely not in sample 2, which is homogeneous and relaxed. Therefore, the driving force for the anisotropic distribution could be the presence of mismatch strain along the $c$-axis due to the formation of the $\mathrm{Ga}$ enriched shell region. The $c$-mismatch strain is in turn due to the peculiar growth mechanism of the core-shell nanowires during which the In enriched core and the $\mathrm{Ga}$ enriched shell are spontaneously formed and grow simultaneously. ${ }^{7}$ Our findings are in agreement with the results of Ref. 4 in which the authors found an anisotropic In distribution in the In second shell coordination. In that case, the number of In-In pairs was higher than what expected for a random In distribution, for the out-ofplane polarisation direction. Interestingly, the samples of 
Ref. 4 were planar epilayers of $\mathrm{InGaN} / \mathrm{GaN}$ with in-plane compressive strain and out-of-plane tensile strain. In our case, we find an anisotropy of opposite sign but also, the strain is opposite compared with Ref. 4. Indeed, the In-rich core is compressed in the growth direction by the Ga-rich shell. It would point to a tendency of In to condensate in the direction of tensile strain. Also in our case, the sample showing this feature of In condensation shows a higher PL efficiency, as for the anisotropic sample of Ref. 4. Although the true nature of localisation in InGaN is still under debate, it is worth pointing out that our results are in agreement with the existence of In-N-In atomic condensates, which have been hypothesized to explain the defect-insensitive emission probability of InGaN materials. ${ }^{1}$ In conclusion, our results establish in the specific case of NWs that enhanced formation of such condensates is correlated to strain as already suggested in the case of InGaN quantum wells. Along with this view the increase of the number of In-In pairs, with respect to the random distribution, in the growth plane of core-shell NWs (sample 1), could be a candidate accounting for carriers localization ${ }^{4}$ and the difference of photoluminescence of samples 1 and $2 .^{7}$

In summary, in the present study we have employed a powerful approach combining XAFS, X-ray RSM, MAD, and $a b$ initio calculations that allowed us to unveal alloy ordering at local atomic scale. This method has general validity and can be applied to other alloys and their 1D and 2D heterostructures.

\section{ACKNOWLEDGMENTS}

We thank the Elettra Synchrotron in Trieste for granting beamtime and the staff of XAFS beamline 11.1 for technical support and assistance. We acknowledge the French CRG (ESRF) and ESRF for granting beam time at beamlines BM2-D2AM and BM30-FAME. C.L. is very grateful to Y. Joly for helping him to use FDMNES code. M.G.P. acknowledges the support of project MAT2009-03074 of
Spanish Ministry of Science and Innovation and is grateful to the Diputación General de Aragón for granting support in the frame of the Researchers Mobility program (Grant No. 224-183). C.L., G.T., B.D., and H.R. acknowledge the French ANR for support (Fidel project ANR 2011 NANO 02902).

${ }^{1}$ S. F. Chichibu, A. Uedono, T. Onuma, B. A. Haskell, A. Chakraborty, T. Koyama, P. T. Fini, S. Keller, S. P. DenBaars, J. S. Speck, U. K. Mishra, S. Nakamura, S. Yamaguchi, S. Kamiyama, H. Amano, I. Akasaki, J. Han, and T. Sota, Nature Mater. 5, 810 (2006).

${ }^{2}$ V. Kachkanov, K. P. O’Donnell, R. W. Martin, J. F. W. Mosselmans, and S. Pereira, Appl. Phys. Lett. 89, 101908 (2006).

${ }^{3}$ V. Kachkanov, K. P. O’Donnell, S. Pereira, and R. W. Martin, Philos. Mag. 87, 1999 (2007).

${ }^{4}$ T. Miyanaga, T. Azuhata, S. Matsuda, Y. Ishikawa, S. Sasaki, T. Uruga, H. Tanida, S. F. Chichibu, and T. Sota, Phys. Rev. B 76, 035314 (2007).

${ }^{5}$ P. R. C. Kent and A. Zunger, Appl. Phys. Lett. 79, 1977 (2001).

${ }^{6}$ J. A. Chan, J. Z. Liu, and A. Zunger, Phys. Rev. B 82, 045112 (2010).

${ }^{7}$ G. Tourbot, C. Bougerol, A. Grenier, M. D. Hertog, D. Sam-Giao, D. Cooper, P. Gilet, B. Gayral, and B. Daudin, Nanotechnology 22, 075601 (2011).

${ }^{8}$ M. Newville, J. Synchrotron Radiat. 8, 322 (2001).

${ }^{9}$ V. Favre-Nicolin, M. Proietti, C. Leclere, N. Katcho, M. Richard, and H. Renevier, Eur. Phys. J.: Spec. Top. 208, 189 (2012).

${ }^{10} \mathrm{H}$. Renevier and M. Proietti, in Characterization of Semiconductor Heterostructures and Nanostructures II, edited by G. Agostini and C. E. Lamberti (Elsevier, Amsterdam, 2012), Chap. 8.

${ }^{11}$ C. Brouder, J. Phys.: Condens. Matter 2, 701 (1990).

${ }^{12} \mathrm{~F}$. Boscherini, in Characterization of Semiconductor Heterostructures and Nanostructures II, edited by G. Agostini and C. E. Lamberti (Elsevier, Amsterdam, 2012), Chap. 7.

${ }^{13}$ A. L. Ankudinov, J. J. Rehr, and S. D. Conradson, Phys. Rev. B 58, 7565 (1998).

${ }^{14}$ F. d'Acapito, F. Boscherini, S. Mobilio, A. Rizzi, and R. Lantier, Phys. Rev. B 66, 205411 (2002).

${ }^{15}$ B. Ravel and M. Newville, J. Synchrotron Radiat. 12, 537 (2005).

${ }^{16}$ L. Schwartz and J. Cohen, Diffraction from Materials (Springer-Verlag, Berlin, 1987).

${ }^{17}$ J. C. Mikkelsen and J. B. Boyce, Phys. Rev. B 28, 7130 (1983).

${ }^{18}$ G. Kresse and J. Hafner, Phys. Rev. B 47, 558 (1993).

${ }^{19}$ G. Kresse and J. Furthmüller, Phys. Rev. B 54, 11169 (1996).

${ }^{20}$ Y. Joly, Phys. Rev. B 63, 125120 (2001). 